\title{
Study of Gyrase-A Gene in Mycobacterium tuberculosis Complex and its Clinical Relevance
}

\author{
Neha Kukreti ${ }^{1}$, Yusra Ahmad ${ }^{1}$, Vijay Kumar $^{2}$, Narotam Sharma ${ }^{2}$, Satish Chandra Nautiyal ${ }^{2}$ \\ ${ }^{1}$ Faculty of Pharmacy, Uttarakhand Technical University, Suddhowala, Dehradun, Uttarakhand, India \\ ${ }^{2}$ Central Molecular Research Laboratory, Department of Biochemistry, Shri Guru Ram Rai Institute of Medical and Health Sciences, Shri Guru Ram Rai University, \\ Dehradun, Uttarakhand, India
}

*Corresponding author: Narotam Sharma, Central Molecular Research Laboratory, Department of Biochemistry, Shri Guru Ram Rai Institute of Medical and Health Sciences, Shri Guru Ram Rai University, Dehradun, Uttarakhand, India, Tel: +91-09557650069; E-mail: sharmanarotam5@gmail.com

Received April 16, 2019; Accepted April 25, 2019; Published May 03, 2019

\begin{abstract}
Objectives: This study is being done to create awareness among the public regarding the routine checkup and if any such disease is diagnosed, proper medication should be started on time to prevent condition of patients before reaching to critical situation.

Methods: A total of 70 patients were included in the study, for the determination of Mycobacterium tuberculosis (MTB) positive and for this mpb64 (Mannose-Binding Protein) gene was targeted at 240 base pair. The samples detected for MTB positive were taken for the determination of gyr-A gene resistance and then samples were undertaken to pre-amplification area for preparing master mix by using forward and reverse primer (5'-GATGACAGACACGACGTTGC-3'and 5'-GGGCTTCGGTGTACCTCAT-3' respectively). After that samples were taken to thermal cycler PCR (Polymerase Chain Reaction) for amplification of gyr-A gene and then amplicons were taken for electrophoresis following the analyzing of amplicons in Electronic UV trans-illuminator system, in which gyr-A gene was targeted at 398 base pair to determine if sample is positive for fluoroquinolone resistance.
\end{abstract}

Results: Males were found highly positive for tuberculosis and gyrase-A resistance. Patients above 41 years were more prone to both tuberculosis and gyrase-A resistance.

Conclusions: Drug resistance is very common among the population which can only be prevented by creating awareness programs resistance.

Keywords: PCR; MTB; Deoxyribonucleic acid (DNA); mpb64 gene; gyr-A gene

\section{Introduction}

DNA topoisomerases are double-stranded and helical in nature [1]. For most of the processes individual DNA strand is required to be separated either temporarily (by transcription), or permanently (by replication).The topological alterations of DNA are catalyzed by topoisomerase enzyme for essential cellular functions [2]. On the basis of mechanism of action, this enzyme is broadly classified as type I and type II [3]. Type II topoisomerase is important nucleic acid that involves in the regulation of DNA topology mostly in regulation of DNA super-coiling [4]. Mycobacterium tuberculosis is causative agent of tuberculosis which possesses only one type II topoisomerase including DNA gyrase-A gene [5]. DNA gyrase-A gene not only supercoils the DNA but also enhances the relaxation, DNA cleavage and decatenation activities. DNA gyrase-A gene enzyme is hetero-tetramer in nature which includes:

i) Subunit A for gyrA gene-consisting two domains, N-terminal breakage-reunion domain and carboxy terminal domain.

ii) Subunit B for $g y r B$ gene-consisting ATPase domain toprim domain [6]. Breakage-reunion domain contains catalytic tyrosine, responsible for the cleavage and re-ligation of the DNA double helix and form DNA duplex [7].

The ATPase domain dimerizes the ATP binding and bind with DNA duplex which cause the transient break in DNA duplex and release the DNA [8]. Fluoroquinolone is a class of synthetic antimicrobial agent and targets the bacterial type II topoisomerase. It shows antibacterial activity by binding with ATPase domain-DNA duplex complex, blocking the DNA replication and cause cell death [9]. Fluoroquinolones are powerful second line drugs that are used for the treatment of Tuberculosis (TB) [10]. Tuberculosis (TB) is an air-borne disease that generally affects the lungs and leads to cough, fever and chest pain [11]. Mycobacterium tuberculosis complex (MTBC) is gram positive bacteria that comprise the M. tuberculosis, M. bovis, M. microti, $M$. africanum, $M$. canetti, and $M$. caprae species which are responsible for tuberculosis [12]. Mycobacterium tuberculosis was discovered by Robert Koch (1843-1910) in 1882 [13]. According to a survey it was found that in India about 2 million people develop active disease each year and half of these can die [14]. Drug resistant tuberculosis is an extreme case of tuberculosis that shows resistance to one or more than one anti-tubercular drugs [15]. Types of drug resistance: Multi-Drug Resistant TB (MDR-TB) is defined as resistance toward at least, isoniazid and rifampicin among first-line drugs. Extensively Drug Resistant TB (XDR-TB) refers to a strain of MTB that are resistant to MDR-TB with at least, any fluoroquinolone and any one of the second-line injectable drugs such as capreomycin, kanamycin and amikacin [16]. Resistance to TB drug is characterized by the types of drug to which the bacteria lack susceptibility and mechanism by which resistance occurred [17]. Resistance to Fluoroquinolones, such as ofloxacin (OFX), mainly occurs by mutation in the target genes gyr-A gene but less frequently in gyrB gene [18].

\section{Methodology}

The research work was carried out at Central Molecular Research Laboratory, Department of Biochemistry, Shri Guru Ram Rai Institute of Medical and Health Sciences (SGRRIM\&HS), Patel Nagar, Dehradun (Uttarakhand). TB positive patients were recruited for the drug resistance study. Clinical specimens were collected from Out Patient Department (OPDs) and In Patient Departments (IPDs) of different Departments of Shri Mahant Indresh Hospital, Dehradun, (Uttarakhand) India and the specimens included were Pulmonary such as Sputum, Pleural fluid, Bronchiolar alveolar lavage, bronchial secretions and extra pulmonary specimens such as Pus, Urine, Semen, Tissue, Endometrial blood, 
Cerebrospinal Fluid for the study. The written consent was taken from patients. Collected samples were undergone for further testing in areas: Bio-safety Cabinet: After collecting the sample from different departments, the isolation of DNA was done by using silica column method. Pre-amplification area: In this area, master mix was prepared for PCR by targeting the specific gene ( $m p b 64$ and gyrase-A gene at 240 and 398 base pair respectively). Amplification area: This area was used for the amplification or multiplication of targeted gene by PCR. Post amplification area: After amplification, the amplicons (final product) were analyzed by using electrophoresis unit.

\section{Results}

The present study was done for the detection of mycobacterium infection in collected pulmonary and extra pulmonary samples of patients. We used PCR as a tool for detecting mycobacterial $m p b 64$ gene and gyrase- $A$ gene resistance in both pulmonary and extrapulmonary samples from suspected TB patients. 70 samples were taken from the different Departments in Shri Mahant Indiresh Hospital and processed in Central Molecular Research Laboratory, Department of Biochemistry, Shri Guru Ram Rai Institutes of Medical and Health Sciences, Patel Nagar, Dehradun, Uttarakhand, India. On the basis of presence of mycobacterium, 2 samples were found positive in the process of AFB (acid fast bacilli) staining and 9 samples were positive for $m p b 64$ gene (TB), while 4 came positive for gyrase-A gene resistance by using PCR. Since the samples belonged to a population of individuals of varying age therefore in order to analyze as to which age group has higher probability of having mycobacterium infection, and for considering some other factors, a complete table has been shown (Table 1). The brief summary of the results are given in the tabular forms also. In Figures 1 and 2 the following data are given; Pus: Pus was $5(7.14 \%)$ of which $1(20 \%)$ was positive and $4(80 \%)$ negative for MTB and $0 \%$ was positive and $1(20 \%)$ was negative for gyrase-A gene. Tissue: $2(2.85 \%)$ of the samples were tissue derived of which $0(0 \%)$ were positive for both MTB and gyrase-A gene and 2(2.85\%) was negative for MTB. Bronchioaveolar Lavage (BAL): BAL were $7(10 \%)$ sample giving $6(85 \%)$ and $4(66.66 \%)$ were positive for both MTB and gyrase-A gene and $1(14.28 \%)$ and $2(33.33 \%)$ were negative for both MTB and gyrase-A gene in TB PCR. Cerebrospinal Fluid (CSF): 27(38.5\%) samples came from CSF and 1(3.7\%) was positive and 26(96.29\%) was negative for MTB while $0 \%$ was positive for gyrase-A gene. Menstrual Blood (MB): Samples from menstrual blood were $1(1.42 \%)$ and $0 \%$ was positive while $1(1.42 \%)$ was negative for MTB. Pleural Fluid (PF): 12(17.14\%) represented PF from which $1(8.33 \%)$ was positive and $11(91.66 \%)$ was negative for MTB while $0 \%$ positive for gyrase-A gene. Ascitic Fluid (AF): $7(10 \%)$ was AF, $0(0 \%)$ positive and $7(10 \%)$ negative for MTB. Pericardial Fluid (PF): PF was $1(1.42 \%)$ and being $0 \%$ positive while $1(1.42 \%)$ negative for MTB. Sputum: Sputum cases with $2(2.85 \%)$ samples; for MTB $0 \%$ coming positive while $2(2.85 \%)$ came negative. Urine: Urine sample were $2(2.85 \%)$ giving $0 \%$ positive and $2(2.85 \%)$ negative for MTB in TB PCR. Synovial Fluid (SF): 1(1.42\%) SF sample was received and $0 \%$ came positive while $1(1.42 \%)$ negative for MTB in TB PCR.

The results from TB PCR indicated that of the 70 clinical samples, grouping the sample according ages, the range was of 20 intervals of age as presented below: $00-20$ years of age were $12(17.14 \%)$ of the processed samples, giving $2(2.87 \%)$ positive and $10(14.28 \%)$ negative results in TB PCR for MTB cases while $0 \%$ was negative to gyrase-A gene resistance (Table 2) (Figure 3). 21-40 years group were $17(24.28 \%)$ of the 70 clinical samples out of which $1(1.42 \%)$ was positive and $16(22.85 \%)$ were negative for MTB cases with $0 \%$ positive to gyrase-A gene resistance. Age range between 41-60 years had the highest number

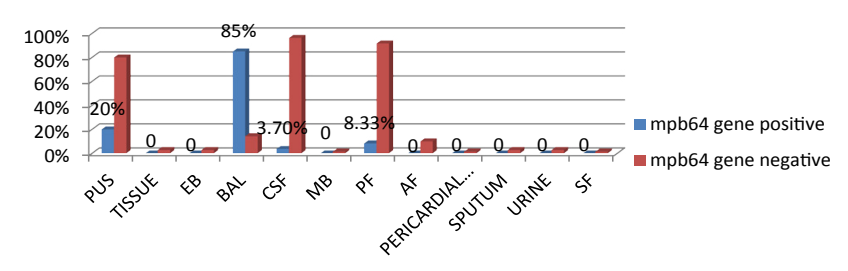

Figure 1: Graph shown sample type vs. mpb64 gene.

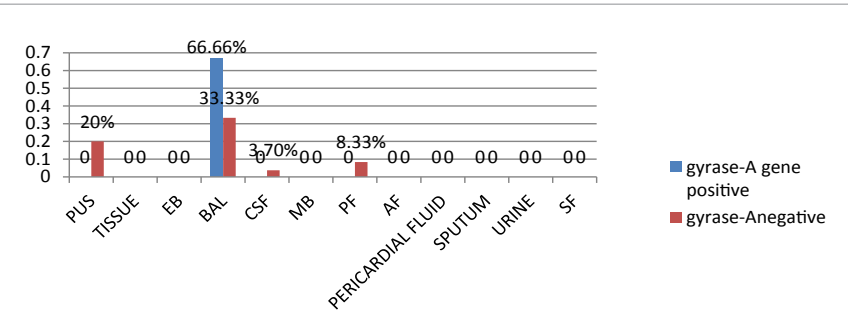

Figure 2: Graph show sample type vs. gyrase-A gene drug resistance relationship.

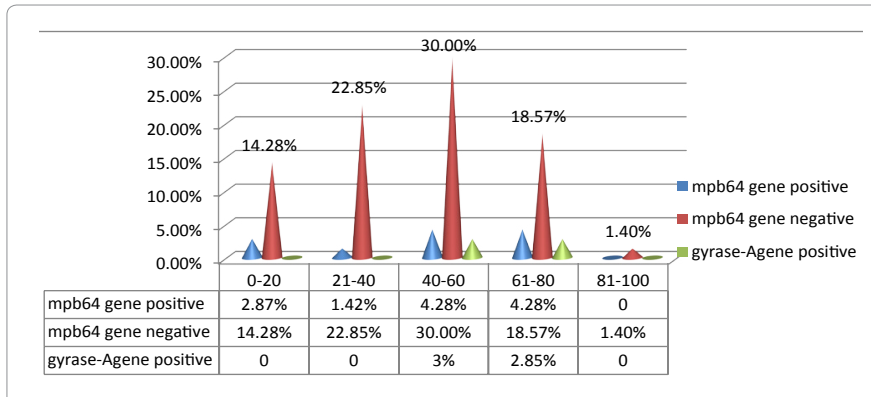

Figure 3: Relationship between ages and $m p b 64$ gene and gyrase- $A$ gene.

of processed clinical samples making $24(34.28 \%)$ of the samples from which $3(4.28 \%)$ being positive for MTB cases, but in gyrase-A gene resistance, $2(3 \%)$ were positive. $16(22.85 \%)$ samples were receive from the age group 61-80 with $3(4.28 \%)$ positive while 21 (18.57) negative for MTB cases whereas $2(3 \%)$ positive to gyrase-A gene resistance. 81 and above of age were $1(1.42 \%)$ of the processed samples, giving $0 \%$ positivity for MTB cases.

As indicated in Table 3 and Figure 4, 30(42.85\%) of the clinical specimens were from females giving $3(10 \%)$ positive and $27(90 \%)$ negative MTB cases while 1(33.33\%)was positive and 2(66.66\%) were negative to gyrase-A resistance. $40(57.14 \%)$ of the clinical specimens were from males giving $6(15 \%)$ positive and $34(85 \%)$ negative MTB cases while $3(50 \%)$ were positive and 3(50\%) were negative to gyrase-A gene resistance.

\section{Discussion}

Tubercle bacilli or Mycobacterium tuberculosis complex (MTBC) is a species of gram-positive bacteria that cause tuberculosis. Tuberculosis (TB) is one of the major causes of morbidity and mortality and its data suggests that there are about 9.6 million new cases and 1.5 million deaths occur annually around the world. In this work, we found that BAL sample was found highly positive for both MTB and gyrase-A gene resistance. The data regarding age group, TB positive cases ranging from $40-60$ and $61-80$ years patients were highly prone to TB and the resistance of gyrase-A gene was highly positive for age group 
Citation: Sharma N, Neha K, Yusra A, Chandra NS (2019) Study of Gyrase-A Gene in Mycobacterium tuberculosis Complex and its Clinical Relevance. Int J Drug Dev \& Res 11: 34-36

\begin{tabular}{|c|c|c|c|c|c|c|}
\hline \multirow{2}{*}{ S.no } & \multirow{2}{*}{ Sample types } & \multirow{2}{*}{ Cases } & \multicolumn{2}{|c|}{ mpb64 gene } & \multicolumn{2}{|c|}{ Gyrase- $A$ gene } \\
\hline & & & Positive & Negative & Positive & Negative \\
\hline 1 & Pus & $5(7.14 \%)$ & $1(20 \%)$ & $4(80 \%)$ & 0 & $1(20 \%)$ \\
\hline 2 & Tissue & $2(2.85 \%)$ & 0 & $2(2.85 \%)$ & 0 & 0 \\
\hline 3 & Endometrial fluid & $2(2.85 \%)$ & 0 & $2(2.85 \%)$ & 0 & 0 \\
\hline 4 & Bronchio-alveolar lavage & $7(10 \%)$ & $6(85 \%)$ & $1(14.28 \%)$ & $4(66.66 \%)$ & $2(33.33 \%)$ \\
\hline 5 & Cerebrospinal fluid & $27(38.5 \%)$ & $1(3.7 \%)$ & $26(96.29 \%)$ & 0 & $1(3.7 \%)$ \\
\hline 6 & Menstural blood & $1(1.42 \%)$ & 0 & $1(1.42 \%)$ & 0 & 0 \\
\hline 7 & Pleural fluid & $12(17.14 \%)$ & $1(8.33 \%)$ & $11(91.66 \%)$ & 0 & $1(8.33 \%)$ \\
\hline 8 & Ascitic fluid & $7(10 \%)$ & 0 & $7(10 \%)$ & 0 & 0 \\
\hline 9 & Pericardial fluid & $1(1.42 \%)$ & 0 & $1(1.42 \%)$ & 0 & 0 \\
\hline 10 & Sputum & $2(2.85 \%)$ & 0 & $2(2.85 \%)$ & 0 & 0 \\
\hline 11 & Urine & $2(2.85 \%)$ & 0 & $2(2.85 \%)$ & 0 & 0 \\
\hline 12 & Synovial fluid & $1(1.42 \%)$ & 0 & $1(1.42 \%)$ & 0 & 0 \\
\hline
\end{tabular}

Table 1: Sample wise positivity and negativity results (Sample data).

\begin{tabular}{|c|c|c|c|}
\hline \multirow{2}{*}{ Age } & \multirow{2}{*}{ Cases } & \multicolumn{2}{|c|}{ mpb64 gene gene } \\
\cline { 2 - 4 } & & Positive & $(10) 14.28 \%$ \\
\hline $0-20$ & $12(17.14 \%)$ & $(2) 2.87 \%$ & $(16) 22.85 \%$ \\
\hline $21-40$ & $17(24.28 \%)$ & $(1) 1.42 \%$ & $(21) 30.00 \%$ \\
\hline $41-60$ & $24(34.28 \%)$ & $(3) 4.28 \%$ & $(13) 18.57 \%$ \\
\hline $61-80$ & $16(22.85 \%)$ & $(3) 4.28 \%$ & 0 \\
\hline
\end{tabular}

Table 2: Age of patients (Age's data).

\begin{tabular}{|c|c|c|c|c|}
\hline \multirow{2}{*}{ Gender } & & mpb64 gene & mpb64gene & Gyrase-A gene-A \\
& Total cases & Positive & Negative & Positive \\
\hline Female & & $10 \%$ & $90 \%$ & $33.33 \%$ \\
\hline Male & $42.85 \%$ & $15 \%$ & $85 \%$ & $50 \%$ \\
\hline
\end{tabular}

Table 3: Gender of patients (Gender's data).

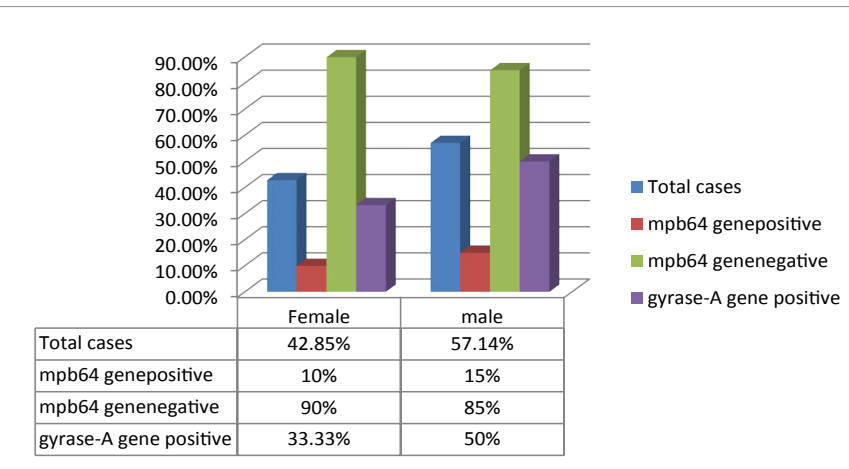

Figure 4: Relationship between gender, $m p b 64$ and gyrase-A gene.

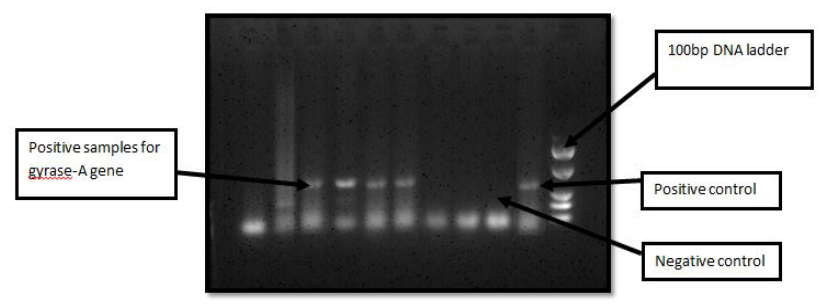

Figure 5: DNA gel electrophoresis for gyrase-A gene.
40-60.The data obtained regarding gender that the Males were more prone to MTB than female. Male showed $15 \%$ positive TB cases while female showed $10 \%$ TB positive response. The resistance to gyrase-A gene in male was also higher than female. Male showed $50 \%$ gyrase-A gene-resistance while female showed $33.33 \%$ gyrase-A gene resistance respectively (Figure 5).

\section{Conclusion}

In recent years the mortality and morbidity rate for tuberculosis has increased due to drug resistance. Early diagnosis of tuberculosis is very important to prevent the patients from drug resistance. Detection of tuberculosis and drug resistance by PCR is rapid technique and it takes approximately 3 hours and gives very accurate result. This study shows that nowadays Fluoroquinolones resistance commonly occurs in the region of Uttarakhand. This could be due to dose irregularity, patients' in-compliances or the drug being prescribed in normal bacterial infections.

\section{Acknowledgment}

The authors are grateful to Honorable Chairman, Shri Guru Ram Rai Education Mission and faculties of Uttarakhand Technical University for their kind support and guidance.

\section{Reference}

1. Vologodskii A (2017) Topology and Physics of Circular DNA. CRC Press.

2. Ventola CL (2015) The antibiotic resistance crisis: part 1: Causes and threats Pharmacy Therapeut 40: 277. 
Citation: Sharma N, Neha K, Yusra A, Chandra NS (2019) Study of Gyrase-A Gene in Mycobacterium tuberculosis Complex and its Clinical Relevance. Int J Drug Dev \& Res 11: 34-36

3. Alexander SP, Fabbro D, Kelly E, Marrion N, Peters JA, et al. (2015) The concise guide to PHARMACOLOGY 2015/16: Enzymes. British J Pharma 172: 6024-6109.

4. Travers A, Muskhelishvili G (2015) DNA structure and function. The FEBS J 282: $2279-2295$

5. Korycka-Machala M, Plocinski P, Dziadek J (2017) Mycobacterial DNA replication as a target for antituberculosis drug discovery. Curr Topics Med Chem 17: 2129-2142.

6. Patel K, Tyagi C, Goyal S, Jamal S, Wahi D, et al. (2015) Identification of chebulinic acid as potent natural inhibitor of $M$. tuberculosis DNA gyrase and molecular insights into its binding mode of action. Comput Biol Chem 59: 37-47.

7. Klostermeier D (2018) Why two? On the role of (a-) symmetry in negative supercoiling of DNA by gyrase. Inter J Mol Sci 19: 1489.

8. Groothuizen FS, Sixma TK (2016) The conserved molecular machinery in DNA mismatch repair enzyme structures. DNA Repair 38: 14-23.

9. Renuka J. Design and biological evaluation of novel Staphylococcus aureus DNA GyrB inhibitors to treat pathogenic infections (Doctoral dissertation, BITS Pilani).

10. Zignol M, Dean AS, Alikhanova N, Andres S, Cabibbe AM, et al. (2016) Population-based resistance of Mycobacterium tuberculosis isolates to pyrazinamide and Fluoroquinolones: results from a multicountry surveillance project. The Lancet Infect Dis 16: 1185-1192.
11. Mohammadi H, Dehghan SF, Golbabaei F, Roshani S, Pakzad R, et al. (2017) Pulmonary functions and health-related quality of life among silica-exposed workers. Tanaffos 16: 60 .

12. Wang HY, Lu JJ, Chang CY, Chou WP, Hsieh JC, et al. (2019) Development of a high sensitivity TaqMan-based PCR assay for the specific detection of Mycobacterium tuberculosis complex in both pulmonary and extrapulmonary specimens. Sci Rep 9: 113

13. Loddenkemper R, Murray JF, Gradmann C, Hopewell PC, Kato-Maeda M (2018) History of tuberculosis. Tuberculosis (ERS Monograph). Sheffield, European Respiratory Society 1: 08-27.

14. Mehta B, Siddiquie A, Kaushik R, Bisht R, Sharma N (2015) Amplification of rpob, kat $g$ \& mab a (fab g1)-inh a promotor dna sequences by pcr in multiple drug resistance tuberculosis. Int J Life Sci Scienti Res 1: 15-18.

15. Van Zyl L, du Plessis J, Viljoen J (2015) Cutaneous tuberculosis overview and current treatment regimens. Tuberculosis 95: 629-638.

16. Tiberi S, Carvalho AC, Sulis G, Vaghela D, Rendon A, et al. (2017) The cursed duet today: Tuberculosis and HIV-coinfection. La Presse Médicale 46: e23-39.

17. Luke EM, Sharma N, Nautiyal SC, Sharma A, Sharma V, et al. (2017) Molecular and microbiological assays for characterization of multi-drug resistant tuberculosis (MDR-TB) in clinical samples-clinical relevance for the disease diagnosis and treatment. Int J Pharma Res Health Sci 5: 1779-1784.

18. Brossier F, Guindo D, Pham A, Reibel F, Sougakoff W, et al. (2016) Performance of the new version (v2. 0) of the GenoType MTBDRsl test for detection of resistance to second-line drugs in multidrug-resistant Mycobacterium tuberculosis complex strains. J Clin Microbiol 54: 1573-1580. 